\title{
Review on Integration of Process PlanNing AND SCHEDUling
}

\author{
PHANDEN, R.K.; JAIN, A. \& VERMA, R.
}

Abstract: Process planning and scheduling are two most important tasks in a manufacturing company. Both functions play an important role in order to deliver the products on time and optimum utilization of resources as well as profitability of manufacturing a product. Several researchers have focussed on the need for integration of process planning and scheduling functions in order to facilitate flexibility and for improving profitability of manufacturing a product, delivery time as well as creation of realistic process plans that can be executed readily on shop floor. In this paper, an integration aspect of process planning and scheduling functions has been introduced. Reported research is classified according to three common integration approaches, non-linear process planning and scheduling, closed- loop process planning and scheduling and distributed process planning and scheduling approaches with their relative advantages and disadvantages. Also, several future research directions have been suggested.

Key words: integration, process planning, scheduling, review, integration of process planning and scheduling
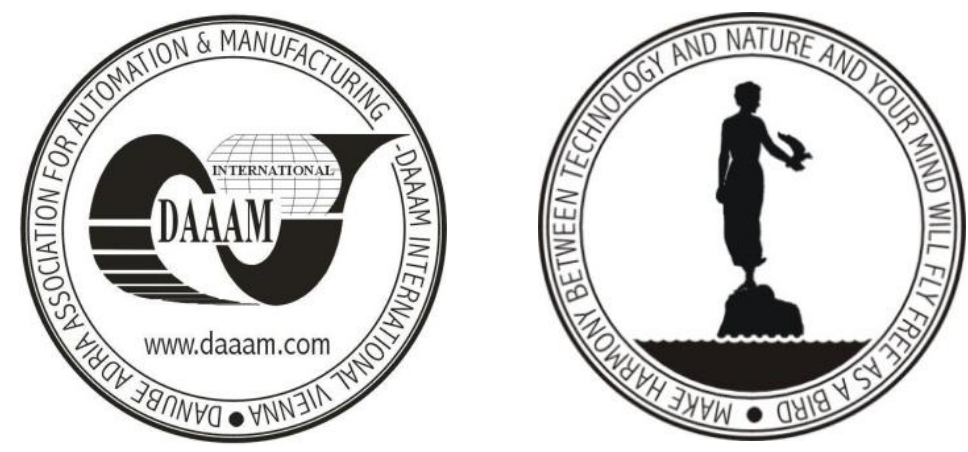

Authors' data: Phanden, R[akesh] K[umar];Assoc. Prof. Jain, A[jai]; Verma, R[ajiv], Department of Mechanical Engineering, NIT Kurukshetra, India, ajayjainfme@nitkkr.ac.in,rakesh_razz@yahoo.com

This Publication has to be referred as: Phanden, R[akesh] K[umar]; Jain, A[jai] \& Verma, R[ajiv] (2011). Review on integration of process planning and scheduling, Chapter 49 in DAAAM International Scientific Book 2011, pp. 593-618, B. Katalinic (Ed.), Published by DAAAM International, ISBN 978-3-901509-84-1, ISSN 17269687, Vienna, Austria

DOI: 10.2507/daaam.scibook.2011.49 


\section{Introduction}

Both, process planning and scheduling play an important role to influence profitability of manufacturing a product, resource utilization and product delivery time (Yang et al., 2001). Process planning can be defined as a systematic determination of methods by which a product is to be manufactured economically and competitively. The process planning function generates process plans, which specifies raw materials needed to produce a product as well as processes and operations necessary to transform raw materials into the final product. Therefore, outcome of process planning is information required for manufacturing processes, including reorganization of machines, tools, and fixtures. On the other hand, in scheduling function a specific task is assign to a specific machine in order to achieve a given performance measure. Moreover, scheduling function is bound by process sequencing instructions that are dictated by process plan and by the time-phased availability of production resources. Thus, both process planning and scheduling involve assignment of resources and are tightly interwoven with each other as well as and optimality of scheduling depends on the results of the process planning. Traditionally, process planning and scheduling are carried out in two distinct and sequential phases, where scheduling is done separately, after the process planning. Moreover, traditional approach is based on concept of subdividing the tasks into smaller and separated duties in order to satisfy the requirements of sub optimization and suitable for mass production (Larsen and Alting, 1992). But, today's manufacturing environment is quite different from traditional one and it's characterized by decreasing lead time, exacting standards of quality as well as larger part variety. In such a complex manufacturing environment, it is difficult to get a satisfactory result using conventional approach due to following reasons: (Larsen and Alting, 1992; Zhang and Merchant, 1993; Gindy et al., 1999; Morad and Zalzala, 1999; Baykasoglu and Ozbakir, 2009; Li et al., 2010a, b and c; Phanden et al., 2011).

The process planner assumes that resources at shop floor are idle and available in unlimited capacities in shop. Therefore, process planner plans for the most recommended resources to processes. This leads to the process planner favouring to select the desirable resources repeatedly. Thus, unrealistic process plan will generate that may not be readily executed on the shop floor.

In sequential approach, fixed process plan restrict the schedule to only one machine per operation. Therefore, possible choices of schedule using alternative machines are ignored.

During process planning the dynamic status of shop is considered, but the constraints considered in planning phase may change greatly because of time delay in between planning phase and scheduling phase. Therefore, generated process plan may become sub-optimal or invalid due to time delay.

Process planning and scheduling focus on single criterion optimisation to determine optimal solution. However, real manufacturing environment involves more than one optimisation criterion. 
Process planning emphasizes on technological requirements while the scheduling focuses timing aspects of it. Thus, conflicting objectives of process planning and scheduling functions are keeping them away.

By considering an integrated approach to process planning and scheduling these deficiencies of traditional approach can be overcome. An integrated approach can respond better than traditional approach to present manufacturing environment and facilitate flexibility, improves profitability of a product, resource utilization, product delivery time and creation of realistic process plans that can readily be executed without frequent alterations (Chryssolouris and Chen, 1985; Sundaram and Fu, 1988; Saygin and Kilic, 1999; Lee and Kim, 2001; Kumar and Rajotia, 2003). Thus, Integration of Process Planning and Scheduling (IPPS) is essential to achieve eventually integrated manufacturing and to dismiss conventional manufacturing approach.

In this paper, various integration models and methods available in literature in the area of IPPS are surveyed and summarised. In conducting this review, some key research challenges are identified. Moreover, different basic IPPS approaches have been discussed with their advantages and disadvantages. Section 2 of the paper presents the various approaches to integration along with related contributions. Section 2.1 discusses Non-Linear Process Planning and Scheduling Approach. Section 2.2 presents Closed-Loop Process Planning and Scheduling Approach. Section 2.3 deals with Distributed Process Planning and Scheduling Approach. Section 2.4 presents Other Approaches for IPPS that are followed by researchers. This literature at the end critically presents the conclusions and some key research directions in section 3 .

\section{Literature Review}

Both, process planning and scheduling are NP - hard, the resulting problem is also NP-hard (Khoshnevis and Chen, 1990). Therefore, it is not suggested to merge both problems to solve it. Moreover, process planning and scheduling department in a company have to be completely dismantled and reorganised. Thus, it cannot be implemented in a company with existing process planning and scheduling departments. The best way to integrate is by increasing information exchange between process planning and scheduling functions. On the basis of this concept several classifications schemes are suggested by various researchers (Zhang and Merchant, 1993; Huang, 1995; Gindy et al., 1999; Gaalman et al., 1999; Zhang et al., 2003a; Shen et al., 2006; Baykasoglu and Ozbakir, 2009; Wang et al., 2009; Guo et al., 2009; Li et al., 2010b). However, present work follows the most commonly used classification among researchers (Larsen and Alting 1990; Zhang and Merchant, 1993; Huang, 1995; Gindy et al., 1999; Gaalman et al., 1999; Baykasoglu and Ozbakir, 2009). Accordingly, there are three main approaches of integration viz., Non-Linear process planning and scheduling Approach, Closed-Loop Process Planning and Scheduling Approach and Distributed Process Planning and Scheduling Approach. These integration approaches are discussed in following section. 


\subsection{Non-Linear Process Planning and Scheduling Approach}

This approach considering operation flexibility (possibility of performing an operation on more than a machine), sequencing flexibility (possibility of interchanging the sequence in which required manufacturing operations are performed) and processing flexibility (possibility of producing the same manufacturing feature with alternative operations, or sequence of operations) in order to generate the multiple process plans of jobs before it enters to shop floor (Benjaafar and Ramakrishnan, 1996). The underlying assumption is that all problems that can be solved ahead of time should be solved before the manufacturing starts. Thus, nonliner approach is based on static shop floor situations (Zhang and Merchant, 1993; Gaalman et al., 1999). Multiple process plans are the ranked according to process planning criterion (such as machining time or production time etc.). After ranking these process plans are stored in a process planning database. The first priority plan is always ready for submission when the job is required and then scheduling makes the real decision. If the first priority plan does not fit well in the current status of shop floor, the second priority plan is provided to scheduling. This procedure is repeated until a suitable plan is identified from already generated process plans. The criteria for decisions are due dates and batch size of order, capacity of workshop and optimization criterion for schedule. In this approach the information flow in one way i.e., from process planning to production planning and thus it may be impossible to achieve full optimal results in integrating the two functions (Kempenears et al., 1996). Moreover, modern production systems maintain MPP (Kim and Egbelu, 1999) and it seems to be a proper means to realise the integration between process planning and scheduling (Kempenears et al., 1996). In addition, it can be implemented in a company with existing process planning and scheduling department. When there are large numbers of parts, the number of process plans tends to increase exponentially and can cause a storage problem (Usher, 2003). Also, some of the process plans created are not feasible according to real time shop status and considering all possible process alternatives for resource allocation may enormously increase the complexity of process plan representation (Zhang and Merchant, 1993; Huang et al., 1995).

The idea of IPPS was first introduced by Chryssolouris et al. (1984), and they proposed Manufacturing Decision Making Approach (MADEMA). They formed a Decision matrix in order to select alternatives, where row represents alternative while column represents attribute and entry was value of attribute for corresponding alternative. Sundaram and $\mathrm{Fu},(1988)$ proposed a scheduling approach in order to minimise makespan as well as to balance loads for machines in a job shop. They used an automated system based on Group Technology (GT) called Integrated ComputerAided Process Planning and Scheduling (ICAPPS) to improve the schedule. Also, a group scheduling algorithm called Key Machine Loading (KML) and combined it with Process Planning Generator (PPG) and Operation Planner (OP) was developed.

Tonshoff et al., (1989) proposed to create all possible process plans for each job before manufacturing starts and suitable process plans are selected according to availability of resources. Shrihari and Greene, (1990) developed a prototype Computer Aided Process Planning (CAPP) for a Flexible Manufacturing System (FMS). GT coding system was used to input information of parts parameters. 
Heuristic knowledge was used to decide sequence of operations and route through system, on basis of the flow-time of jobs.

Jablonski et al., (1990) proposed a three module scheme called Flexibly Integrated Production planning and Scheduling system (FIPS). First was an automated feature recognition module, second was a Process Planning Module (PPM) and third was a scheduling and dispatching module. Authors concluded that flexible and reactive scheduling approach on feature based process planning was feasible.

Palmer, (1996) proposed a Simulated Annealing (SA) based approach, which contained three types of configuration alterations; (i) reverse the order of two sequential operations on a machine, (ii) reverse the order of two sequential operations within a job, and (iii) change the method used to perform an operation. The cost functions such as, tardiness, mean flow time, makespan and a combined function of mean flow time and tardiness were considered. Authors concluded that solution quality of SA was remaining high across varying situations and it was effective means for IPPS.

Kim and Egbelu, (1999) presented a mathematical approach for multiple jobs with each having multiple process plans in a job shop. They claimed that the proposed method minimize makespan for part mix. The scheduling problem was solved with two algorithms viz., Pre-processing algorithm and Heuristic/Iterative algorithm. "Pre-processing algorithm" combined features of branch and bound and integer programming techniques while heuristic/iterative algorithm combined features of branch and bound technique and Earliest Completion Time (ECT) dispatching rules. Authors concluded that computational time of pre-processing algorithm was substantially lower than that of mix integer programming technique but higher than that of heuristic model. As number of jobs increases, solution quality obtained by heuristic got worse, but as number of machine increases it had no clear effect on performance of heuristics.

Aldakhilallah and Ramesh, (1999) proposed a structure called ComputerIntegrated Process Planning and Scheduling (CIPPS) which contained three modules viz., Super Relation Graph (SRG), Cover Set Model (CSM) and Cover Set Planning and Scheduling Module (CSPS) in order to recognise polyhedral depression features and extract prismatic features from CAD database using Artificial Neural Network (ANN) and computational geometry techniques. Moreover, CIPPS framework contained three modes of operations viz., Dynamic Support for Design Decision (DSDD), runtime Intelligent Operational Control (IOC) and Data Consolidation and Integration (DCI). DSDD mode supports decisions during design process. IOC mode worked up for automatic shop floor management, when changes occurred in environment.

Weintraub et al., (1999) presented a method in order to minimize manufacturing cost while satisfying due dates by taking into account alternative process plans of jobs, in a large scale manufacturing job shop. They developed an iterative simulation-based scheduling algorithm to minimize lateness and applied in Virtual Factory, which was a Windows based software package. In order to further reduce lateness, a Tabu-Search (TS) based algorithm was applied to identify process plans with alternative operations and routings. The numbers of alternative process 
plans were fixed for the job at two. Process plans with alternative routings, operations and sequences were selected according to current shop status. Authors concluded that scheduling with alternatives can greatly improves the ability to satisfy due dates under varying shop status.

In Saygin and Kilic, (1999) an integrated approach, that utilise multiple process plans with off-line scheduling in an FMS was proposed. It works in four stages: (i) Machine tool selection (ii) Process plan selection (iii) Scheduling and (iv) Rescheduling Module. Authors concluded that idle time of machine tool was reduced to 30 units from 81units and waiting time of parts was dropped to 50 units from 74 units. Moreover, best system performance is not assured by a optimal process plan having shortest processing time or least number of operations.

In Lee and Kim, (2001), simulation based on genetic algorithm (GA) was proposed to solve IPPS problem. Simulation module computes performance measures based on process plans combination created by GA instead of process plan alternatives and output the near-optimal process plan combination prior to execution on shop floor. Authors concluded that about $20 \%$ reduction of makespan was possible when compared with random selection of process plan combination.

In Yang et al., (2001) the process plan was generated directly from part design and available resource data information. The system had four components viz., a relational manufacturing database, form feature recognition, process alternative generation, and scheduling state evaluation. A 3D model and blank raw material model was entered by using initial graphics exchange specification (IGES) data format. The manufacturing features were decomposed by using graph-based and rulebased algorithm. They concluded that proposed prototype contained choice of process sequence with verification of delivery time for all feasible set of process sequences.

Moon et al., (2002) developed an IPPS model based on GA for Multi-plant Supply Chain (MSC). Operations Sequencing Problem (OSP) was formulated as a multiple Travelling Salesman Problem (TSP's) and each TSP determine machine operation sequences for each part type. A Topological Short Technique (TST) was used to obtain all flexible sequences in directed graph. They concluded that proposed GA approach was more efficient than TS approach in terms of computational time and problem size.

Kim et al., (2003) proposed a technique called Symbiotic Evolutionary Algorithm (SEA) to simultaneously deal with process planning and job shop scheduling. SAE was based on the fact that parallel searches for different pieces of solution were more efficient than a single search for the entire solution. Authors considered operation flexibility, sequencing flexibility and processing flexibility during process planning. The job-shop scheduling determines both process plan for each job and corresponding schedule, while optimising two types of objectives: minimising makespan and minimising mean flow time.

Kumar and Rajotia, (2003) proposed a method for on-line scheduling in a CAPP system for a job shop. A scheduling factor was used to make operationmachine assignment. The scheduling criterions were flow-time and number of tardy jobs. They concluded that the proposed method helps in on-line determination and assignment of loads on various machines. Further, Kumar and Rajotia, (2006) 
presented a scheme for integration model for job-shop. It considered machine capacity and cost while assigning operation to machines. The proposed framework contained two controlling modules viz., process plan generator and scheduler. Both modules were interacting with a Decision Support System (DSS). DSS interacts with various databases such as machine tool database, tool-work material database and machining parameters database. A generative scheme was used in order to develop process plan for axis-symmetric components.

Zhao et al., (2004) presented an approach for IPPS based on GA for a job shop. A Fuzzy Inference System (FIS) was used to select alternative machines. It was based on Fuzzy Logic toolbox by MATLAB. Based on capability of machines, GA was used to balance load for all machines. The scheduling objectives were to minimise makespan, minimise number of rejects and minimise processing cost. Zhao et al., (2006) extended their earlier work and used Particle Swarm Optimisation (PSO) algorithm for balancing load on each machine. Moreover, Zhao et al., (2010) proposed an IPPS applicable to Holonic Manufacturing System (HMS) in which they used a hybrid PSO and Differential Evolution algorithm in order to balance the load for all machines.

Grabowik et al., (2005) proposed a methodology represented a predictivereactive and event-driven approach to rescheduling. They utilised multiple process plans of a product to respond in disturbances during manufacturing. Authors concluded that availability of processes routes expanded flexibility of control system and increases efficiency of rescheduling.

Choi and Park, (2006) proposed a GA based method for IPPS, that minimise makespan of production order, considering alternative machines and alternative operations sequences in integrated manufacturing. An operation-based representation was used to construct chromosomes. The performance of proposed method was evaluated in a job shop evolving multiple process plans.

Jain et al., (2006) proposed an IPPS scheme that can take advantage of flexibility on the shop floor and can be implemented in a company with existing process planning and scheduling departments. The proposed scheme was able to take advantage of MPP while following a real time strategy for scheduling suitable for changing workshop status. It composed of two basic modules: Process Plans Selection Module (PPSM) and Scheduling Module (SM). PPSM selects best four process plans for each part type and stores them in a database. SM performs part scheduling for using best four process plans. The effectiveness of MPP over single process plan was assessed through makespan and mean flow time. They concluded that availability of multiple process plans during scheduling of manufacturing system can reduce mean flow time and makespan.

Li and McMahon, (2007) discussed an IPPS system based on SA for a job shop. Processing, operation sequencing and scheduling flexibility were used to explore search space. The performance measures were makespan, balanced level of machine utilisation, job tardiness and manufacturing cost. The algorithm was defined in two sets of data structures viz., first set represents process plans and second set specify the schedule of a group of parts. Authors concluded that proposed algorithm performed satisfactory and was able to choose one or more performance criterion in 
order to address practical requirements. Also, the proposed algorithm was compared with GA, TS and PSO algorithms.

Moon et al., (2008) presented an evolutionary search method based on TST for IPPS in supply chain. A mixed integer programming model was formulated, which incorporate process planning of resources selection and sequence of operation as well as determination of their schedule to optimise makespan.

Li et al., (2008b) presented a GA-based approach to facilitate IPPS. Authors developed an efficient genetic representation and operator scheme. The first part of chromosomes was composing of alternative process plan string and second part was scheduling plan string. They considered a job shop in order to minimise makespan. Authors found that value of makespan without integration was worse than proposed integration model. Li et al., (2010c) proposed a hybrid approach which synthesizes advantage of GA and TS to solve IPPS problem. The first part of chromosome was alternative process plan string; second part was scheduling plan string and third was machine string. Third part selects machine set of corresponding operations of all jobs to minimise makespan. They concluded that proposed algorithm was effective and acceptable for IPPS.

Wang et al., (2008) developed an IPPS procedure for a batch manufacturing environment by utilising process plan solution space. A heuristic was developed to minimise tardiness as well as to maintain cost of process plan involved in modification of process plan. Authors concluded that Process Planning Module optimises the route with minimum processing cost; therefore the tardiness of jobs was improved while the cost of process plan was maintained at low level.

Haddadzade et al., (2009) discussed an IPPS approach for a job shop for prismatic components and it took advantage of MPP to fulfil due dates using overtime. It contains two modules viz., PPM and SM. PPM generates all possible alternative plans then SM ranked these based on minimum cost while due date was considered. The proposed approach can optimise cutting parameters for milling operations only. They concluded that proposed approach can determine machining parameter, tool, machine and amount of overtime within minimum cost objective and due date.

Baykasoglu and Ozbakir, (2009) proposed an IPPS approach that a Generic Process Plan (GPP) generator to generate final process plan and a dispatching rule based heuristic to generate feasible schedules. A Multiple Objective Tabu Search (MOTS) algorithm was employed for two objectives that were "flow time" and "cost of process plan". They concluded that as the process plan flexibility increases process plan cost decreases. In Rajkumar et al., (2010) a multi-objective Greedy Randomised Adaptive Search Procedures (GRASP) was presented in order to minimise makespan, maximum workload, total workload, tardiness and total flow time evolving flexible job-shop environment. It consists of two phase viz., construction phase and local search phase. Proposed approach was consisted of PPSM and SM. They concluded that proposed approach was effective in order to solve IPPS problem.

Leung et al., (2010) utilising Ant Colony Optimisation (ACO) algorithm based on Multi-Agents System (MAS) to minimise makespan for IPPS in job shop. They considered processing flexibility of alternative routing and alternative machines. 
AND/OR graphs were used to represent MPP. They concluded that proposed approach was able to solve IPPS problem.

\subsection{Closed-loop Process Planning and Scheduling Approach}

In this approach, dynamic feedback from production scheduling and status of available resources is considered in order to generate process plans. The scheduling phase tells process planning regarding availability of different machines on shop floor for the coming job, so that every plan is feasible with respect to current availability of production facilities. Every time an operation is completed on shop floor, a featurebased work piece description is studied in order to determine next operation and allocate the resources. Dynamic behaviour of the manufacturing system is considered in Closed Loop Process Planning and Scheduling Approach. Thus, real time status is crucial for Closed Loop Process Planning and Scheduling Approach (Zhang and Merchant, 1993). This approach is also called dynamic approach or real time approach. In order to take full advantage of Closed-loop Process Planning and Scheduling Approach, process planning and scheduling departments in a company may have to be dismantled and reorganized (Iwata and Fukuda,1989). Also, it requires high capacity hardware and software and adaptation of step-by-step local view limits the solution space for subsequent operations (Zhang and Merchant, 1993; Gaalman et al., 1999). However, Closed Loop Approach is unrealistic as the complexity of manufacturing processes might be unavoidable in achieving real-time process plan generation (Joo et al., 2001).

Dong et al., (1992) proposed an IPPS approach in which product features were extracted using an AI-based feature extractor with respect to shop floor conditions. Then, rough process plan for a product was prepared. It considers all possible manufacturing ways for each operations volume (operation features) that can be produced in one machine setup considering shop floor capabilities. A rough process plan with alternative was input to scheduling.

COncurrent Manufacturing PLANning and shop control for batch production (COMPLAN), a European ESPRIT project 6805 during the period 1992-1995, integrates process planning and workshop scheduling using MPP. The goal of COMPLAN project was to develop a software prototype that was capable of carrying out manual and automatic process planning and scheduling based on MPP, in a small batch manufacturing in a job shop. It was an extension of FLEXPLAN (Kruth and Detand, 1992). COMPLAN consists of PPM and Workshop Scheduling System (WSS). PPM was capable of handling MPP and it could use projected resources load while developing MPP. Also, PPM module described feasible manufacturing alternative that provided flexibility to workshop scheduling.

Usher and Fernandes, (1996) presented an approach called Process planning ARchitecture for Integration with Scheduling (PARIS). It used feature based approach to planning and has two phases namely "Static Planning" and "Dynamic Planning". "Static Planning" phase involved selection, assignment, and sequencing of processes and machines that exist within the shop. The output of "Static Planning" phase was a set of alternative macro-level plans. "Dynamic Planning" phase considered availability of shop floor resources and objectives specified by scheduler. 
The proposed approach was feasible to perform for both prismatic and rotational parts. They concluded that the proposed approach was able to reduce work load when real time portion of planning activities were carried out in second phase.

Cho et al., (1998) developed a prototype Block Assembly Process Planning and Scheduling (BAPPS) system in shipbuilding which consists of a block assembly PPM, a SM, a bottleneck block selection module and a process re-planning module. Rule-based reasoning technology was applied to determine optimal assembly units and assembly sequences in generating initial process plans. For SM, a schedule revision heuristic was developed for efficient reallocation of blocks to alternative assembly shops. For bottlenecks, block selection which plays cardinal role in bridging process planning and scheduling, a heuristic was developed by employing an entropy-based partitioning method to identified bottleneck periods.

Sugimura et al., (2001) proposed an IPPS model in which process planning phase selected suitable sequence of machining feature using GA approach. This model was based on HMS. The optimum sequence of machining equipment was selected using Dynamic Programming (DP) after taking into consideration the future schedule of machining equipments, with objectives to minimise total machining and set-up time. Machining schedules were determined using a real-time scheduling procedure in which, individual job selected suitable machining equipment, in order to carry out next operation based on process plan. Sugimura et al., (2003) extended their earlier work (Sugimura et al., 2001). Here, optimum sequence of machining equipment, two objective functions viz., minimization of shop time and machining cost were used in DP approach. Shop time was combination of machining time, fixturing time, tool changing time, transportation time and waiting time. The machining time was calculated on the basis of manufacturing process time and operation cost per unit time of manufacturing resources. They concluded that the proposed approach was feasible to select both suitable sequence of machining equipment and machining schedule concurrently.

Shrestha et al., (2008) proposed an IPPS system utilising DP method based modification of process plans. Two types of holons viz; job holons and scheduling holons were considered for process planning and scheduling respectively. Based on feasible process plans of all jobs, the scheduling holon generates the schedule of all equipments. Makespan, total machining cost and weighted tardiness cost were assumed as an objective function for scheduling. A GA based method was adopted for selecting a combination of process plans. Schedules were generated using a set of dispatching rules viz; SPT, SPT/Total Work Remaining and Apparent Tardiness Cost (ATC). Feedback processes were considered in order to modify the process plans based on load balancing of machining equipments. Two approaches viz; centralised approach and distributed approach were developed in order to modify process plans. In distributed approach, the job holons modify their process plans without any centralised control of scheduling holons. In centralised approach, the feedback information from scheduling holon after scheduling was transferred to job holons and one modified set of process plans obtained with consideration of constraints of machining equipments. They concluded that in centralised approach of process plan 
modification, the makespan was improved for the case where there was concentration of the machining load on some machining equipments.

Zhang et al., (2003a) discussed an IPPS system similar to COMPLAN except, the process planning system could generate whole solution space based on operations for a given part using SA and GA in order to find optimal plan (Kempenears et al., 1996). This approach was developed for batch manufacturing of prismatic parts. An "Intelligent facilitator" was used to generate instructions for process plan modifications. The integration was achieved through an "intelligent facilitator" that provide feedback to PPM of a particular job. Also, they developed two algorithms viz.; "machine-utilisation" and "tardy-job" based on heuristic and concluded that algorithms based on heuristic were suitable to achieve a satisfactory schedule. Wang et al., (2008) have extended the work of Zhang et al., (2003a). They proposed two heuristic algorithms namely fine-tuning (FH-Tardy) and quick-tuning (QH-Tardy). In FH-tardy algorithm, solution space of selected operation of selected tardy job was modified in each iteration. In QH-Tardy algorithm, solution space of selected operation was modified in each iteration for each tardy job. Authors concluded that job tardiness was reduced utilising the proposed heuristic that can explore process plan solution space.

Wong et al., (2006a) developed an agents-based multi-stage negotiation protocol scheme for IPPS in a job shop. It contained part agents and machine agents to represent parts and machines respectively. Part and machine agents negotiate to establish schedule using process plans and operation details from AND/OR graph. The negotiation protocol was able to handle multiple task and many-to-many negotiation. A currency conversion function, which incorporates processing time and due-date, was adopted for bidding. A java-based simulation model Multi-Agents Negotiation (MAN) was used to implement the proposed approach. Authors concluded that in the pursuit of local objectives such as parts flow time, the proposed approach performs better than meta-heuristics. Wong et al., (2006b) extended their earlier work (Wong et al., 2006a) and proposed a hybrid-based multi-agent system called Online Hybrid Agents-based Negotiation (oHAN). It was consists of local agents (part and machine agents) and a supervisor agent. The supervisor agent was used for global rescheduling process and influenced the decisions made by local agents. It acted as a system coordinator manager in between local agents for global rescheduling objective. They concluded that hybrid approach is effective for larger scale rescheduling and provided a better global performance. Authors introduced a mobile agent in oHAN in order to handle job orders.

\subsection{Distributed Process Planning and Scheduling Approach}

Here, both process planning and production scheduling performs simultaneously. It divides process planning and production scheduling tasks into two phases. The first phase is preplanning and second phase is the final planning phase. In the first phase, process planning function analyzes the job based on the product data. The features and feature relationships are recognized, and corresponding manufacturing processes are determined. The required machine capabilities are also estimated. Second phase matches required job operations with the operation 
capabilities of available production resource. The integration occurs at the point when resources are available and the job was required. The result is dynamic process planning and production scheduling constrained by real time events. This approach is also called as phased or progressive approach or just-in-time approach. This approach is the only one that integrates the technical and capacity-related planning tasks into a dynamic fabrication planning system (Larsen and Alting, 1990). However, this approach requires high capacity and capability from both hardware and software. Also, scope of Distributed Process Planning and Scheduling Approach is limited within some specific CAPP functions such as process and machine selection as detailed process planning tasks are shifted down to manufacturing stages for enhancing flexibility. From implementation viewpoint both process planning and scheduling departments in a company have to be dismantled and reorganised (Joo et al., 2001; Haddadzade et al., 2009).

Aanen et al., (1989) proposed a hierarchical approach for IPPS in which the primary objective was to satisfy due-dates of the order and secondary was to minimize change-over and idle times of machines within time horizon. Initially, planning function was solved and resulting output becomes the input for scheduling. Feedback information was provided to planning level if output of scheduling was not satisfied. They planned and scheduled two types of activities viz., machining activities and operator activities. The time horizon (of about 10 days) at the planning level was divided in periods of one day. For each day, machining activities to be performed were assigned. The resulting list was called a day list. List of activities for first day was the input to scheduling level. Scheduling criterion was to minimise makespan of the day list. Initially, the scheduling of machining activities was performed, and then the scheduling of operators activities. Scheduling of machining activities is performed using branch-and-bound method.

Zhang and Merchant, (1993) developed an Integrated Process Planning Model (IPPM). It contains three levels of integration viz., pre-planning module at initial integration level, pairing planning module at decision making level and finalplanning module at functional integration level. Pre-planning module performed three activities viz., feature reasoning, process recognition and setup determination. The output of pre-planning was possible setups, machining operation and associate times. Pairing planning module worked in three steps: machining selection, tooling and fixture selection and exact time selection. Final planning level worked in three steps viz., operational tolerance analysis, operation sequencing and overall time and cost calculations. In pre-planning level, SM provides available equipment in next time window. In decision making level, the available equipments were matched with requirement of setup and matching processes. Decision Making Module (DMM) was central element to perform by means of real time information.

Huang et al., (1995) presented a progressive approach containing three phases namely pre-planning, pairing planning and final planning. The activities within each phase takes place in different time periods. Pre-planning was executed in early stage, as soon as product design finished. Pairing Planning executed in later stage, when an order has been released to the shop and final planning was executed just before the manufacturing begins. The interaction between process planning and scheduling take 
place in all three phases. The model composed of PPM and SM. PPM was responsible for generating process plans according to part design specifications. The criterion for process plan selection was manufacturing lead time. SM was responsible for allocating resources in the shop and overall management of flow of production orders. Authors solved IPPS problem by developing first mathematical models and then using optimisation algorithms.

Kempenears et al., (1996) discussed a collaborative process planning and scheduling system. It was based on (i) the aim of "Evaluation Module" (EM) to improve quality of delivery work, and (ii) the idea of "feedback" at all levels. Feedback information was used by evaluation modules to improve the quality of delivered output. It consists of Workshop Evaluator (WE), Schedule Evaluator (SE) and Process Plan Evaluator (PPE). WE captured the performance information of workshop that was total operation time, machine breakdown and problems with process plans etc. Workshop disruptions were recorded and appropriate measures were taken. SE evaluates performance of schedule versus results of workshop. A request can be launched to add an alternative to specific process plan to bypass a resource that has broken down. PPE takes feedback data into account, and it deals with production constraints coming from SE. The feedback loop from scheduling to process planning was based on the established production constraints. A set of constraints, express the demand from scheduling concerning quality of operation routings. "General Constraints" considered current and predicted loading of shop, while in "Specific Constraints", the scheduler asks process planning department to regenerate process plan.

Mamalis et al., (1996) developed an on-line IPPS system in which first phase was an offline process planning and schedule generation and second decision phase was an on-line process planning and scheduling in order to consider the disruption at shop floor. Here, information flow exists, from and into the CAPP and scheduling system. It provides dynamic feedback to process planning system. On the basis of feedback, MPP were generated by process plan generator. A Decision Making Module (DMM) react to modifications of the initial production conditions and provides optimal scheduling decisions, on the basis of variable routing and dispatching strategies such as first-come-first-serve (FCFS), SPT and longest processing time (LPT). The objective was to minimise operation time during process planning and minimisation of total delay of parts during scheduling. They designed an information model to maintain and develop an interaction between process planning and scheduling module. The proposed approach was validated using simulation in job-shop scheduling for machining of rotational parts.

Gu et al., (1997) presented a bidding based MAS approach that has four types of agents namely; part, shop manager, machine and tool. The machine agents performs process planning, that includes, STEP parsing and interpretation (to produce data for process planning), tolerance analysis, operations and setup planning, machines, tools and fixtures selection whereas other agents performs the standard functions related with the resources to which they represents. The scheduling was based on the cost model. It makes decisions during the process of negotiations, with 
consideration of machining time, setup time, tool change time, cost of tooling and due date of part.

Sadeh et al., (1998) developed an Integrated Process Planning/Production Scheduling (IP3S) shell based on a blackboard architecture that supports concurrent development and dynamic revision of integrated process planning scheduling solutions. It consists of a blackboard, a controller, a collection of knowledge sources - including a process planning knowledge sources, a production scheduling knowledge sources, a communication knowledge sources and several analysis knowledge sources (e.g., a knowledge source to generate resource utilization statistics and to evaluate resource contention in different situations) - and a Motif-based graphical user interface (GUI).

Morad and Zalzala, (1999) presented an approach using concept of CE with GA in order to simultaneously optimise processing capabilities of machines including processing costs as well as number of rejects in alternative machines with scheduling of jobs. The chromosome consists of order of parts as well as order of operations and associated machines to perform operations. The formulation was based on multiobjective weighted-sums optimisation. The objectives were to minimise total rejects produced, to minimise total cost of production and to minimise makespan and weight for total rejects produced, total cost of production and makespan were taken as w1, w2 and w3 respectively. The scheduling was based on SPT dispatching rule. They compared their weighted sum approach with traditional and Multi Objective Genetic Algorithm (MOGA) approach. Authors concluded that conventional method performed worst then weighted sum approach for improvement of makespan and total number of rejects produced.

Gindy et al., (1999) presented an IPPS approach based on Concurrent Engineering (CE). It contains a knowledge-base facility modelling functions, a feature-based process planning system and a simulation-based SM. Resource Element (RE) concept was used for representation of manufacturing environment. It described process capability, which contained information of commonality and uniqueness of machines inside factory of Form Generating Schema (FGS). FGS was a combination of cutting tool of specific geometry, a set of relative motions between a part and cutting tool and technological output. During process planning, a number of alternatives using Technological Solutions for Feature (TSF) were produced for each feature of a part. Machine tool of manufacturing system was described by a set of REs. A machine-independent generic process plan was input for scheduling system in order to optimise machine utilisation and tardiness. Final process plan and production schedule were generated simultaneously just before the part was going to be manufactured. Authors compared performance of machine-based (conventional) system and RE based proposed system on the basis of mean tardiness and average flow time. They concluded that proposed approach was much less sensitive to dispatching rules and due date assignment approaches.

Chan et al., (2001) developed an MAS-based scheme for integrated, distributed and co-operative process planning system called IDCPPS. It works at three levels viz., initial planning level, decision making level and detail planning level. Initial planning level involved: features reorganisation, selection of machining processes, 
generation of process sequences, and manufacturability evaluation. The result of this level was a set of alternative process plans. Decision making level was interacted with scheduling system in order to consider availability of shop floor resources. The result of this level was a set of ranked near-optimal alternative process plans. Detailed planning level included: tool selection, final machine selection, machine parameters determination, calculation of estimates for both machining cost and time. The output of this level was the final detailed linear process plans. The integration with scheduling was considered at each stage with process planning. They concluded that IDCPP system was a tool for CAD by integrating down-stream constraints into the design phase.

Wu et al., (2002) presented an IPPS scheme for a Digital Virtual Manufacturing (DVM) environment using Java-based MAS. A cost function was proposed for optimal partner selection in virtual enterprise, which considered partner's manufacturing capability, processing time, partners location and part due date. The framework was divided into two phases' viz., enterprise phase and partner phase. Enterprise phase selected optimal manufacturing partner in a DVM and second phase performed at shop floor-level integration. They concluded that cost function found to be effective for optimal partner selection in a DVM.

Wang et al., (2003) proposed an approach based on design-for-machining concept termed as 'Distributed Process Planning' (DPP). The design was based on two-layer structure for supervisory planning and operation planning. It had three major sub systems for design, planning and control, which shared one dynamic database. A real-time network and secured factory network enabled the functionality of each module. They concluded that the dynamism of process planning enabled by DPP methodology is crucial to next-generation reconfigurable manufacturing system (RMS), and it is beneficial to apply DPP to the RMS framework. Also, the IPPS can be done early at supervisory planning level where machine selection was conducted.

Zhang et al., (2003b) developed an IPPS system called Concurrent Integrated Process Planning System (CIPPS). It can integrate CAD, process planning and scheduling through working of process planning and scheduling system in parallel at three levels viz., initial planning level; decision making level and detailed planning level. They concluded that CIPPS suits the requirements of agile manufacturing such as response to changing conditions of organisation; integration with different domains of manufacturing and reduction in time and cost of processes.

Wang \& Shen, (2003) discussed the agent-based decision making, machining feature-based process planning and function-block based process execution associate with DPP. An example of pocket milling in order to generate a function-block based process plan was presented. Moreover, Wang et al., (2005) proposed a framework of collaborative process planning supported by a real-time monitoring system. Authors concluded that an adaptive process plan of the part can be generated by converting its machining features to appropriate function blocks designs. Details on function-block design in DPP can be found in Wang et al., (2006). Wang et al., (2009) presented a function-block technology based adaptive approach for design and integration of event-driven function-blocks with process/set-up planning and execution monitoring. 
In Wang, (2009) presented an IPPS system for web-based collaborative planning and control, supported by real-time monitoring for dynamic scheduling.

Zattar et al., (2008) developed MAS for IPPS in a job shop. It utilised a feature-based time-extended negotiations protocol for decision-making about realtime adaptation of process plan with alternatives in order to minimise makespan and mean flow time. The total operation time as reported in Kim et al., (2003) was divided in three parts: $20 \%$ processing time in the machine; $70 \%$ machine setup and $10 \%$ fixturing setup. Authors found that mean makespan and mean flow time was 426.68 minutes and 329.59 minutes respectively, without consideration of setup time while, the values of mean makespan and mean flow time was 374.46 minutes and 286.96 minutes with consideration of setup time. They concluded that global average values of makespan and flow time obtained by the proposed approach were better than those generated by Symbiotic Evolutionary Algorithm (SEA) of (Kim et al., 2003).

Zattar et al., (2010) extended their earlier work (Zattar et al., 2008). Total operation time as reported in (Kim et al., 2003) was divided in three parts: $80 \%$ processing time in the machine; $10 \%$ machine setup and $10 \%$ fixturing setup. They found that mean improved rate of makespan and flow time was $3.66 \%$ and $9.13 \%$ respectively, without consideration of setup time while, the values of mean improved rate of makespan and flow time was $6.18 \%$ and $10.56 \%$ with consideration of setup time. They concluded that reduction of both objectives due to reduction in number of machines changes on which the jobs were manufactured.

Cai et al., (2009) presented an IPPS methodology indirectly through a multimachine setup planning approach using GA, which utilised the adaptability of process plan associated with setups. A tool accessibility examination approach was used for adaptive setup planning (ASP) and it was extended to solve multi machines setups planning problem. They concluded that GA-based ASP is capable to quickly respond in changing shop floor situations.

Wang et al., (2010) proposed an IPPS approach for job shop machining operations via a two-step ASP using GA's. It consists of generic setup planning (step one) and adaptive setup merging (step two) in order to optimise cost, quality, makespan and machines utilisation. Initially, three-axis based setup plans was created and then merge some setups in order to create final setup with consideration of available machines. Final specific process plan was created after scheduling and setup merging. They concluded that based on machines availability and capability the proposed approach can generates setup plans adaptively.

Ueda et al., (2007) presented an approach to produce simultaneous decision of process planning and scheduling utilising Evolutionary Artificial Neural Network (EANN). The local decisions such as selection of part to process and selection of machine for selected part were taken by machine agents. Each machine learns the state of shop floor using EANN. System objective was achieved by multiplications of result of each machines learning.

Li et al., (2008a) proposed a Cooperative Process Planning and Scheduling system (CPPS) for IPPS in a job shop. The system equipped with three Game theory strategies viz., Pareto strategy, Nash strategy and Stackelberg strategy in order to 
minimise makespan, job tardiness, manufacturing cost and load balancing of machines. Three algorithms were applied to CPPS problem viz., PSO, SA and GA. Authors considered machine breakdown and new order arrival as dynamic features of a job shop. They concluded that lower manufacturing cost can be achieved through utilisation of cheap machines, but it is conflicting with the criterion of balanced level of machine utilisation. Moreover, SA-based algorithm took shorter time to find good solution but it was dependent on its parameters and the problem to be optimised.

Shukla et al., (2008) presented a bidding based MAS in which tool cost was considered as dynamic quantity rather than a constant. Tool cost comprises tool-using cost and tool repairing cost. Tool cost was predicted by data-mining agent. When a job arrives at shop floor, the component agent announces a bid for one feature at a time to all machines agents. Once all features were assigned to appropriate machine, this information was utilised by optimisation agent to find optimal process plan and schedule using hybrid TS-SA algorithm.

Zhanjie \& Ju, (2008) discussed an IPPS approach in which process route was selected on the basis of balanced level of machines utilisation, minimum processing cost and SPT dispatching rules. Authors found that for $70 \%-80 \%$ of test-bed problems, as reported in (Kim et al., 2003) proposed GA provided the best performance among the algorithm compared.

Li et al., (2009, 2010a) presented an agent-based approach for IPPS with an optimisation agent and a mathematical model for IPPS in a job shop. This approach contained three agents and databases. Job agents and machine agents were used to optimise alternative process plan and schedule. All three flexibilities namely operational, sequential and processing flexibility were used in process planning. When changes occur at shop floor and determined schedule can't be carried out, machine agents negotiate with other agents (including job agents and optimisation agents) in order to trigger a rescheduling process. The objective of process planning system was to minimise production time whereas for scheduling system it was to minimise makespan. In Li et al. (2011) a game theory based hybrid algorithm has been applied to solve the multi-objective IPPS problem.

\subsection{Other approaches}

Some of the IPPS approaches available in the literature are not belongs to any of the above category. These approaches are presented below.

Liao et al., (1993) developed a Computer Managed Process Planning (CMPP) in order to achieve CAPP/Scheduling integration. Process Decision Model File (PDMF) and Machine Tool File are the primary functional areas of CMPP which should be modified for CAPP/Scheduling integration. PDMF contained process decision rules in order to determine process plans. An operation-machine index was developed for selecting best machine from all alternates in process planning stage to satisfy two scheduling criterion, first was to minimise mean flow time and second was to reduce number of jobs tardy. The modifications were: (i) the creation of a Secondary Machine Tool File containing data needed to calculate operation-machine index, and (ii) a software program for modifications of process decision rules. 
Authors concluded that total processing time was reduced by $6 \%$ resulting from the use of altered PDMF.

Zijm \& Kals, (1995) developed and implemented an IPPS system in a small batch manufacturing shop in which a set of initial process plans and a resource decomposition procedure was used to determine schedule that minimize lateness. If, schedule performed unsatisfactory, a critical path analysis was conducted to select jobs as candidates for MPP's. The critical path graph automatically selects an operation which causes the largest lateness. The procedure and algorithms were implemented in a multi-resources shop floor planning and scheduling system, called "Job-planner". The system contained three layers viz., an Automatic Scheduler, an Interactive Scheduling Mode \& Monitoring and Control System. "Job-planner" attempted to minimise lateness by calculating paths for each possible schedule.

Chan et al., (2006) presented an Artificial Immune System (AIS) based algorithm inherited with Fuzzy Logic Controller (FLC) to solve IPPS problem. The proposed algorithm could handle multiple orders involving outsourcing strategy. They considered manufacturing system with alternative operations sequences, alternative machines for different operations and precedence relationship between the operations. The system was modelled as Travelling Salesman Problem (TSP) with precedence relationship. The feasible operation sequence was generated by merging features of AIS-FLC, directed graph and topological sort techniques. The objective function was to minimise makespan and simultaneously considering due date of customer orders. The proposed algorithm was tested with five machines with one outsourcing machine. Authors concluded that the proposed concept of outsourcing strategy is advantages, only when the total transportation time involved during the process is less than waiting time of the part. The outsourcing strategy effectively reduces makespan. Chan et al., (2009) extended their earlier work (Chan et al., 2006) and proposed an Enhanced Swift Converging Simulated Annealing (ESCSA) algorithm for scheduling. The proposed algorithm was compared with other optimisation methods such as GA, SA, TS and TS-SA algorithms and found that makespan came out 30 and 55 units for due date $\leq 45$ and $\leq 75$ respectively which outperformed comparatively.

Guo et al., (2009) presented a re-planning method for machine breakdown status and new order arrival. The solutions were encoded into PSO particles to search for best sequence of operations through optimisation strategies of PSO algorithm. They concluded that PSO outperformed both GA and SA by considering computational efficiency. The proposed algorithm showed improvement in performance by applying crossover operator taken from GA.

Li \& Ierapetritou, (2009) reviewed various integration methodologies and uncertainties for planning and process scheduling in the process industries. Authors concluded that integration of planning and scheduling and systematic considerations of uncertainties have a tremendous impact on industries in order to increase their production flexibilities.

Shao et al., (2009) presented an IPPS approach based on GA in which process planning and scheduling system were working simultaneously by synthesizing integration methodology of non-linear and distributed approach. A was used. The 
objective of process planning was to minimize production time. However, two objective functions of scheduling were considered. First was to minimise makespan and second was synthesis consideration of makespan and balanced level of machine utilization.

Sormaz et al., (2010) suggested a model for integration of product design with process planning and scheduling information in real time using XML based data representation. It involves features mapping from CAD file, process selection for part design and integration with scheduling and simulation of FMS model utilising alternative routings. Two scenarios viz; integration of CAPP and scheduling and integration of CAPP with FMS control simulation were considered in order to demonstrate the proposed model on a group of nine prismatic parts. Features based model was created in Unigraphics CAD package. Rule-based system was used for process selection and selection of alternative machine available with tool and processing time information. Scheduling application used LP approach for simultaneous selection of alternatives for each feature and their scheduling. FMS simulation model was developed as a discrete event model in Arena. A feature focused dynamic model was developed to integrate all three modules of feature mapping, process planning and FMS controller. The simulation model collects the current machine utilisation level and queue size for every machine and sends this data to FMS controller.

\section{Conclusions}

This paper has presented a review of IPPS approaches and key concepts as well as relative strength and weakness of the three main integration approaches. Integration of process planning and scheduling plays a cardinal role for performance improvement in a manufacturing system. With considerations of recommendation of different approaches reviewed in the present work there are some future research directions have been suggested below.

Adaptive-Initiative and Interactive IPPS model: Generally, two types of disturbances occurs on shop floor viz., internal and external. Most of previous research has not incorporated these shop floor disturbances in the IPPS model. An internal disturbance involves arrival of new machine, routine maintenance, tool failure and machines breakdown. Whereas external disturbances involves order cancellations, rush order arrival and change in demand pattern. Occurrence of internal and/or external disturbances will probably make the current schedule infeasible. Thus, in order to minimise the effects of shop floor disturbances there is a need to develop an adaptive-initiative and interactive IPPS model.

Multi-agents based IPPS Model: In Distributed Process Planning and Scheduling Approach, Multi-agents system approach is found to be the most promising as they are recognised as an effective way to realize IPPS adaptiveness. Agents based approach may perform better when number of agents and level of negotiations is less. However, these approaches may fail to perform when number of agents involved as well as level of negotiations is large (i.e. system size is large) as they have a limited effective negotiation mechanism. In such scenario, there is need 
to increase the capability of supervisor agent so that effective negotiation among resources agents can be achieved.

Multi-objective IPPS Model: Most of the researchers considered single objective to solve IPPS problem. Single objective are not able to represents real manufacturing environment completely. Thus, there is a need to consider multiobjective such as makespan, mean flow time, tardiness of parts simultaneously as multi-objective represents real manufacturing environment better than single objective. Although few studies have considered multi-objectives but more investigations are needed in this aspect.

Implementation of IPPS Model: Most of reported research has not focussed on the development of industrial applicable IPPS system. During the real manufacturing there exist several practical issues such as machine and tool changes, job releasing. Tool failures constraints and subassembly levels of each job, set up time, transportation time, finite buffers and other resources constraints.

Hybrid optimisation based IPPS Model: Most of the previous research utilised an optimisation algorithm. However, as the search space is larger, computation time increases dramatically. Thus, there is a need to develop hybrid optimisation approach that can find optimal solution quickly. In this direction a combination of heuristics and meta-heuristics techniques will be helpful.

At the end we like to lift an important issue i.e. to develop an IPPS approach that can be implemented in a company with existing process planning and scheduling departments and that can integrate process planning and scheduling quickly. This may be done by combining various approaches such as Non Linear Process Planning and Scheduling Approach - Closed Loop Process Planning and Scheduling Approach, Non Linear Process Planning and Scheduling Approach - Distributed Process Planning and Scheduling Approaches so that the developed approach is able to take advantage of combining approaches. This may results in an improved level of information exchange between process planning and scheduling departments. Currently, we are working on the development of an IPPS model of encompassing the features of two approaches viz. Non Linear Process Planning and Scheduling Approach and Closed Loop Process Planning and Scheduling Approach. We hope that the present work will provide a platform to new researchers.

\section{Acknowledgements}

This research is supported by Govt. of INDIA (GOI), Science and Engineering Research Council (SERC), Department of Science and Technology (DST) New Delhi, INDIA. (SR/SR3/MERC-098/2007).

\section{References}

Aanen, E., Gaalman, G.J. \& Nawijn, W.M., (1989). Planning and scheduling in an FMS. Eng. Costs Prod. Econ., 17 (1-4), 89-97 
Aldakhilallah, K. A. \& Ramesh, R., (1999). Computer-Integrated Process Planning and Scheduling (CIPPS): intelligent support for product design, process planning and control, Int. J. of Prod Res, 37(3), 481-500

Baykasoğlu, A. \& Özbakır, L., (2009). A grammatical optimization approach for integrated process planning and scheduling. J. of Intell. Manuf., 20(2), 211221

Benjaafar, S. \& Ramakrishnan, R., (1996). Modeling, measurement and evaluation of sequencing flexibility in manufacturing systems. Int J Prod Res, 4(5), 11951220

Cai, N., Wang, L., \& Feng, H.Y., (2009). GA-based adaptive setup planning toward process planning and scheduling integration. Int J of Prod Res, 47 (10), 27452766

Chan, F., Kumar, V. \& Tiwari, M., (2006). Optimizing the performance of an integrated process planning and scheduling problem: an AIS-FLC based approach', 2006 IEEE Conference on Cybernetics and Intelligent Systems, in Proceedings, 7-9 June, Bangkok, Thailand, 1-8

Chan, F., Kumar, V. \& Tiwari, M., (2009). The relevance of outsourcing and leagile strategies in performance optimization of an integrated process planning and scheduling model. Int J of Prod Res, 47(1), 119-142

Chan, F., Zhang, J. \& Li, P., (2001). Modelling of integrated, distributed and cooperative process planning system using an agent-based approach', Proceeding Institution Mechanical Engineering, Part B: Journal of Engineering Manufacturing, 215, 1437-1451

Cho, K.K., Oh, J.S., Ryu, K.R. \& Choi, H.R., (1998). An Integrated Process Planning and Scheduling System for Block Assembly in Shipbuilding. Annals of the CIRP 47(1), 419-422

Choi, H. \& Park, B., (2006). Integration of process planning and job shop scheduling using genetic algorithm. 6th WSEAS International Conference on Simulation, Modelling and Optimization, in Proceedings, 22-24 September, Lisbon, Portugal, 13-18

Chryssolouris, G. \& Chan, S., (1985). An integrated approach to process planning and scheduling. Annals of the CIRP, 34(1), 413-417

Dong, J., Jo, H.H. \& Parsaei, H.R., (1992). A feature-based dynamic process planning and scheduling. Comp. \& Ind. Eng. 23, 141-144

Gaalman, G.J.C., Slomp, J., \& Suresh, N.C., (1999). Towards an integration of process planning and production planning and control for flexible manufacturing systems. Inter J of F M S, 11, 5-17

Gindy, N., Saad, S. \& Yue, Y., (1999). Manufacturing responsiveness through integrated process planning and scheduling. I J of Prod Res, 37 (11), 23992418

Grabowik, C., Kalinowski, K., \& Monica, Z., (2005). Integration of the CAD/CAPP/PPC systems, Journal of Materials Processing Technology, Volumes 164-165, AMPT/AMME05 Part 2, 15 May, 1358-1368

Gu, P., Balasubramanian, S., \& Norrie, D., (1997). Bidding-based process planning and scheduling in a multi-agent system. Comp \& Ind Eng, 32(2), 477-496 
Guo, Y. W., Li, W. D., Mileham, A. R. \& Owen, G. W., (2009). Optimisation of integrated process planning and scheduling using a particle swarm optimisation approach. International Journal of Production Research, 47(14), 3775- 3796

Haddadzade, M., Razfar, M.R. \& Farahnakian, M., (2009). Integrating process planning and scheduling for prismatic parts regard to due date. In Proceedings of World Academy of Science, Engineering and Technology, No.51, 64-66

Huang, S.S., Zhang, H.C., \& Smith, M.L., (1995). A progressive approach for the integration of process planning and scheduling. IIE Trans, 27,456-464

Iwata, K. \& Fukuda, Y., (1989). A new proposal of dynamic process planning in machine shop. In Proc CIRP International workshop on Computer aided process planning, Hanover University, Germany, 21-22 Sept 1989

Jablonski, S., Reinwald, B. \& Ruf, T., (1990). Integration of process planning and job shop scheduling for dynamic and adaptive manufacturing control. Paper presented at the IEEE, 444-450

Jain, A., Jain, P. \& Singh, I., (2006). An integrated scheme for process planning and scheduling in FMS. Inter. J of Adv Manuf Tech, 30, 1111-1118

Joo, J., Park, S., \& Cho, H., (2001). Adaptive and dynamic process planning using neural networks. Int J Prod Res 39(13), 2923- 2946

Kempenaers, J., Pinte, J. \& Detand, J., (1996). A collaborative process planning and scheduling system. Advances in Engineering Software, 25, 3-8

Khoshnevis, B. \& Chen, Q., (1990). Integration of process planning and scheduling functions. J. Intell. Manuf., 1,165-176

Kim, K. \& Egbelu, P., (1999). Scheduling in a production environment with multiple process plans per job. Inter. J. of Prod. Res., 37(12), 2725-2753

Kim, Y., Park, K. \& Ko, J., (2003). A symbiotic evolutionary algorithm for the integration of process planning and job shop scheduling. Computers and Operations Research, 30, 1151-1171

Kruth, J.P. \& Detand, J., (1992). A CAPP System for Nonlinear Process Plans, CIRP Annals - Manufacturing Technology, 41(1), 489-492

Kumar, M. \& Rajotia, S., (2003). Integration of scheduling with computer aided process planning. Journal of Mate. Proc. Techn, 138, 297-300

Kumar, M. \& Rajotia, S., (2006). Integration of process planning and scheduling in a job shop environment. Inter. J. of Adv. Manuf. Tech., 28(1-2), 109-116

Larsen, N.E. \& Alting, L., (1990). Simulations engineering within process and production planning. Pacific Conf. on Manuf., Australia, 17-21 Dec. 1990

Larsen, N.E. \& Alting, L., (1992). Dynamic planning enriches concurrent process and production planning. Int J Prod Res, 30(8), 1861-1876

Lee, H. \& Kim, S., (2001). Integration of process planning and scheduling using simulation based genetic algorithms. Inter. J. of Adv. Manuf. Tech., 18,586590

Leung, C. W., Wong, T. N., Mak, K. L., \& Fung, R. Y., (2010). Integrated process planning and scheduling by an agent-based ant colony optimization. Comput. \& Ind. Eng., 59(1), 166-180 
Li, W. \& McMahon, C., (2007). A simulated annealing-based optimization approach for integrated process planning and scheduling. Inter. J. of Computer Integrated Manufacturing, 20(1), 80-95

Li, W., Gao, L., Li, X. \& Guo, Y., (2008a). Game theory-based cooperation of process planning and scheduling. CSCWD2008, in Proc., Xi' an, China, 841845

Li, X., Gao, L., \& Li, W., (2011) Application of game theory based hybrid algorithm for multi-objective integrated process planning and scheduling. Expert Systems with Applications, doi:10.1016/j.eswa.2011.07.019

Li, X., Gao, L., Zhang, C. \& Shao, X., (2010b). A review on Integrated Process Planning and Scheduling. Int. J. Manuf. Res., 5(2), 161-180

Li, X., Gao, L., Zhang, G., Zhang, C. \& Shao, X., (2008b). A genetic algorithm for integration of process planning and scheduling problem. Lecture Notes in Artificial Intelligence, 5315, 495-502

Li, X., Li, W., Gao, L., Zhang, C., \& Shao, X., (2009). Multi-agent based integration of process planning and scheduling. In 13th International Conference on Computer Supported Cooperative Work in Design, 215-220

Li, X., Shao, X., Gao, L. \& Qian, W., (2010c). An effective hybrid algorithm for integrated process planning and scheduling, I. J. of Prod. Econ., 126(2), 289298

Li, X., Zhang, C., Gao, L., Li, W., \& Shao, X., (2010a). An agent-based approach for integrated process planning and scheduling. Exp. Sys. with App., 37, 12561264

Li., Z. \& Ierapetritoua, M., (2009). Integration of Planning and Scheduling and Consideration of Uncertainty in Process Operations, Computer Aided Chemical Engineering, 10th International Symposium on Process Systems Engineering: Part A. 27, 87-94, DOI: 10.1016/S1570-7946(09)70235-4

Liao, T.W., Coates, E.R., Aghazadeh, F., Mann, L. \& Guha, N., (1993). Modification of CAPP systems for CAPP/scheduling integration, Comp. \& Ind. Eng, 25(14), 203-206

Mamalis, A.G., Malagardis, I. \& Kambouris, K., (1996). On-line integration of a process planning module with production scheduling. International Journal of Advance Manufacturing Technology, 12, 330-338

Moon, C., Kim, J. \& Hur, S., (2002). Integrated process planning and scheduling with minimizing total tardiness in multi-plants supply chain. Computers and Industrial Engineering, 43, 331-349

Moon, C.,Lee, Y.H., Jeong, C.S. \& Yun, Y.S., (2008). Integrated process planning and scheduling in a supply chain, Comp. \& Ind. Eng., 54(4), 1048-1061

Morad, N. \& Zalzala, A., (1999). Genetic algorithms in integrated process planning and scheduling. J. of Intell. Manuf., 10,169-179

Palmer, G., (1996). A simulated annealing approach to integrated production scheduling. J. of Intell. Manuf., 7,163-176

Phanden, R.K., Jain, A., \& Verma, R., (2011). Integration of process planning and scheduling: a state-of-the-art review, International Journal of Computer Integrated Manufacturing, Volume 24, Issue 6, 2011, Pages 517 - 534 
Potter, M.A., (1997). The design and analysis of a computational model of cooperative coevolution. Ph.D. dissertation, George Mason University, USA

Rajkumar, M., Asokan, P., Page, T., \& Arunachalam, S., (2010). A GRASP algorithm for the integration of process planning and scheduling in a flexible job-shop. International Journal of Manufacturing Research, 5 (2), 230-251

Sadeh, N., Hildum, D., Laliberty, T., McANulty, J., Kjenstad, D. \& Tseng, A., (1998). A blackboard architecture for integrating process planning and production scheduling. Concurrent Engineering: Research and Applications, 6(2), 88-100

Saygin, C. \& Kilic, S., (1999). Integrating flexible process plans with scheduling in flexible manufacturing systems. Inter. J. of Adv. Manuf. Tech., 15,268-280

Shao, X., Li, X., Gao, L. \& Zhang, C., (2009). Integration of process planning and scheduling: a modified genetic algorithm-based approach. Computers and Operations Research, 36, 2082-2096

Shen, W., Wang, L. \& Hao, Q., (2006). Agent-based distributed manufacturing process planning and scheduling: a state-of-the-art survey. IEEE Trans. on Systems, Man, and Cybernetics-Part C: Appl. and Reviews, 36(4), 563-577

Shrestha, R., Takemoto, T., Ichinose, K., \& Sugimura, N., (2008). A study on integration of process planning and scheduling system for holonic manufacturing with modification of process plans. International Journal of Manufacturing Technology and Management, 14 (3-4), 359-378

Shukla, S., Tiwari, M. \& Son, Y., (2008). Bidding-based multi-agent system for integrated process planning and scheduling: a data-mining and hybrid tabu-SA algorithm-oriented approach. Inter. J. of Adv. Manuf. Tech., 38,163-175

Sormaz, D.N., Arumugam, J., Harihara, R.S., Patel, C., \& Neerukonda, N., (2010). Integration of product design, process planning, scheduling, and FMS control using XML data representation. Robotics and Computer-Integrated Manufacturing, DOI:10.1016/j.rcim.2010.07.014

Srihari, K. \& Greene, T.J., (1990). MACRO-CAPP: a prototype CAPP system for an FMS. Int. J. Adv. Manuf. Technol., 5, 34-51

Sugimura, N., Hino, R. \& Moriwaki, T., (2001). Integrated process planning and scheduling in holonic manufacturing systems. IEEE Inter. Symp. on Assembly and Task Planning Soft Res., in Proc,, Park, Fukuoka, Japan,4,250-254

Sugimura, N., Shrestha, R. \& Inoue, J., (2003). Integrated Process Planning and Scheduling in Holonic Manufacturing Systems - Optimization Based on Shop Time and Machining Cost. Proc. of the 5" IEEE International Symposium on Assembly and Task Planning , July 10-11, Besanqon, France, 36-41

Sundaram, R.M. \& Fu, S.S., (1988). Process planning and scheduling. Computer and Industrial Engineering, 15, 296-307

Tan, W. \& Khoshnevis, B., (2000). Integration of process planning and scheduling a review. Journal of Intelligent Manufacturing, 11, 51-63

Tonshoff, H.K., Beckendorff, U. \& Andres, N., (1989). FLEXPLAN: A concept for intelligent process planning and scheduling. CIRP International Workshop, September, Hannover, Germany, 21-22 
Ueda, K., Fuji, N. \& Inoue, R., (2007). An emergent synthesis approach to simultaneous process planning and scheduling. Annals of CIRP, 56(1), 463466

Usher, J. \& Fernandes, K., (1996). Dynamic process planning - the static phase. Journal of Materials Processing Technology, 61, 53-58

Usher, J., (2003). Evaluating the impact of alternative plans on manufacturing performance. Computers and Industrial Engineering, 45,585-596

Wang, J., Zhang, Y. F., Nee, A. Y. C., Wang, Y. F. \& Fuh, J. Y. H., (2009). Reducing tardy jobs by integrating process planning and scheduling functions. International Journal of Production Research, 47(21), 6069-6084

Wang, L. \& Shen, W., (2003). DPP: An Agent-Based Approach for Distributed Process Planning. Journal of Intelligent Manufacturing, 14(5), 429-439

Wang, L., (2009). Web-based decision making for collaborative manufacturing. International Journal of Computer Integrated Manufacturing. 22(4), 334-344

Wang, L., Cai, N. \& Feng, H. (2009). Function blocks enabled dynamic set-up dispatching and execution monitoring. International Journal of Computer Integrated Manufacturing, 22(1), 3-12

Wang, L., Cai, N., Feng, H. Y. \& Ma, J., (2010). ASP: An Adaptive Setup Planning Approach for Dynamic Machine Assignments. IEEE Transactions on Automation Science and Engineering, 7(1), 2-14

Wang, L., Feng, H.Y., \& Cai, N., (2003). Architecture design for distributed process planning. Journal of Manufacturing Systems, 22(2), 99-115

Wang, L., Jin, W., \& Feng, H. Y., (2006). Embedding Machining Features in Function Blocks for Distributed Process Planning. International Journal of Computer Integrated Manufacturing, 19(5), 443-452

Wang, L., Song, Y., \& Shen, W., (2005). Development of a function block designer for collaborative process planning. In Proceedings of the Ninth International Conference on Computer Supported Cooperative Work in Design, 1, 217-222

Wang, Y.F., Zhang, Y.F., Fuh, J.Y.F., Zhou, Z.D.,Lou, P. \& Xue, L.G., (2008). An Integrated Approach to Reactive Scheduling Subject to Machine Breakdown. Proc. of IEEE Inter. Conf. on Auto. \& Log., 1-3 Sep., Qingdao, China, 542-547

Weintraub, A., Cormier, D., Hodgson, T., King, R., Wilson, J. \& Zozom, A., (1999). Scheduling with alternatives: a link between process planning and scheduling. IIE Transactions, 31, 1093-1102

Wong, T., Leung, C., Mak, K. \& Fung, R., (2006b). Integrated process planning and scheduling/ rescheduling- an agent-based approach. International Journal of Production Research, 44(18-19), 3627-3655

Wong, T.N., Leung, C.W., Mak, K.L. \& Fung, R.Y.K., (2006a). An agent-based negotiation approach to integrate process planning and scheduling, International Journal of Production Research, 44(7), 1331-1351

Wu, S., Fuh, J. \& Nee, A., (2002). Concurrent process planning and scheduling in distributed virtual manufacturing. IIE Transactions, 34, 77-89

Yang, Y., Parsaei, H. \& Leep, H., (2001). A prototype of a feature-based multiplealternative process planning system with scheduling verification. Computers and Industrial Engineering, 39,109-124 
Phanden, R.K.; Jain, A. \& Verma, R.: Review on Integration of Process Planning ...

Zattar, I., Ferreira, J. E., Rodrigues, J. G. G.\& De Sousa, C. B., (2010). A multi-agent system for the integration of process planning and scheduling using operationbased time-extended negotiation protocols. International Journal of Computer Integrated Manufacturing, 23(5), 441-452

Zattar, I., Ferreira, J.E., Rodrigues, J.G.G. \& De Sousa, C.B., (2008). Integration between process planning and scheduling using feature-based time-extended negotiation protocols in a multi agent system. Int. J. Services Operations and Informatics, 3(1), 71-89

Zhang, H.C., \& Merchant, M.E., (1993). IPPM - A Prototype to Integrate Process Planning and Job Shop Scheduling Functions. CIRP Annals - Manufacturing Technology, 42(1), 513-518

Zhang, J., Gao, L., Chan, F. \& Li, P., (2003b). A holonic architecture of the concurrent integrated process planning system. Journal of Materials Processing Technology, 139,267-272

Zhang, Y., Saravanan, A. \& Fuh, J. (2003a) 'Integration of process planning and scheduling by exploring the flexibility of process planning', International Journal of Production Research, Vol. 41, No. 3, pp.611-628

Zhanjie, W \& Ju, T., (2008). The Research about Integration of Process Planning and Production Scheduling Based on Genetic Algorithm. CSSE, International Conference on Computer Science and Software Engineering, 1, 9-12

Zhao, F., Hong, Y. and Yu, D. \& Yang, Y., (2004). A genetic algorithm based approach for integration of process planning and production scheduling. Inter. Conf. on Intell. Mecha. and Auto, in Proc., Chengdu, China, 483-488

Zhao, F., Hong, Y., Yu, D., Yang, Y. \& Zhang, Q., (2010). A hybrid particle swarm optimisation algorithm and fuzzy logic for process planning and production scheduling integration in holonic manufacturing systems. International Journal of Computer Integrated Manufacturing, 23(1), 20-39

Zhao, F., Zhu, A., and Ren, Z., \& Yang, Y., (20060. Integration of Process Planning and Production Scheduling Based on A Hybrid PSO and SA Algorithm. Proc. of IEEE Inter. Conf. on Mecha. and Auto. June 25-28, Luoyang, China

Zijm, W.H.M. \& Kals, H.I.J., (1995). The Integration of Process Planning and Shop Floor Scheduling in Small Batch Part Manufacturing. CIRP Annals Manufacturing Technology, 44(1), 429-432 